\title{
Exploring the Field in Your Backyard: Community-based Learning in Environmental Anthropology
}

\author{
David Fazzino \\ College of Liberal Arts, Bloomsburg University of Pennsylvania, USA
}

Copyright $(2016$ by authors, all rights reserved. Authors agree that this article remains permanently open access under the terms of the Creative Commons Attribution License 4.0 International License

\begin{abstract}
This paper discusses the integration of applied and community-based anthropological fieldwork into programs that do not specifically address applied work. While there has been an increased interest in applied work, it is also the case that not all programs and departments are moving to an applied approach. Instructors who attempt to add these as essential components into advanced level classes may face a multitude of daunting challenges, amongst them the ability to balance this with substantive in-class content delivery, meeting regulatory requirements with the institutional review board, varying levels of student knowledge in methods and theory, and attaining and maintaining student interest. This paper discusses a variety of approaches to meet these challenges and suggests a timeline for a 15-week course that achieves a balancing act of substantive course delivery and hands-on experiences, offering students the best of "both worlds".
\end{abstract}

Keywords Undergraduate Training and Teaching, Applied Anthropology, Environmental Anthropology

\section{Overview}

"The map is not the territory" and indeed the view from the classroom is never the view from the field. Progress has certainly been made since the days of Kroeber's sage advice of buying a notebook and a pencil in preparation for fieldwork [1], yet, I suppose that many academic anthropologists still struggle with ensuring that undergraduate students at our institutions have at least a cursory understanding of our discipline's methods. Academic anthropologist may also struggle to ensure that students grasp the broad brush strokes of anthropological theory. Add to this the desire to incorporate application and community-based work and there may be concern that the basics will not be covered if we attempt to do much. Instructors who attempt to add these as essential components into advanced level classes may face a multitude of daunting challenges, amongst them the ability to balance this with substantive in-class content delivery, meeting regulatory requirements with the institutional review board, varying levels of student knowledge in methods and theory, and attaining and maintaining student interest. I will begin by discussing two approaches to applied work in anthropology and then move into a discussion of some of the challenges that I am familiar based on teaching classes which have incorporated aspects of applied and community-based anthropology. I will offer some suggestions to meet these challenges for achieving the balancing act of theory, methods, and hands-on work, thereby offering students the best of academic and applied approaches to anthropology. I will conclude by offering a timeline (Table 1) that I have recently implemented in a course I taught with some slight modifications that will ensure all students are able to effectively grasp and apply what they are leaning in the classroom. It is my hope that this timeline and accompanying discussion will provoke further discussions on these essential issues in undergraduate anthropology training.

\section{Applied Anthropology in American Anthropology}

To me, there appear to be two schools of thought in American Anthropology regarding applied anthropology: the first is that all of the knowledge that we acquire can be applied in one way or another, with the volition of the anthropologist or without. In these settings there is no need for an applied approach or track as it is implied in the mere process of acquiring knowledge and disseminating it. This dissemination can take place in a variety of venues [2], nevertheless so long as it is shared, contemplated, and put into action it meets the requisite indicators of being "applied". The idea that all anthropology is applied anthropology eliminates the need to consider applied work as anything other than part and parcel of the order of things in a discipline which swings at holism and integration.

The second approach follows the proposition that there is something unique in application and in the intent of the anthropologist or anthropologist-in-training, in the case of 
undergraduate students. That is, although knowledge of the human condition, in a broad, holistic, comparative, and evolutionary sense may be utilized at some point for specific purposes, it is not applied until the anthropologist applies the knowledge in unison with selected local actors. At the same time a certain malady of detachment and non-association has permeated our discipline, wherein academics recuse themselves from the ramifications of their research. As Appaudari [3] notes the place and voice of those that we work with are held subservient to academic conventions. He was speaking of issues of place and voice within our discipline. Turner [4] has laid out a challenge for anthropologists to stop playing the academic game and rather enter into conversations with one another and with those outside of our discipline, not only to the extent that we can work in interdisciplinary teams to confront the challenges of the current moment, the contemporary Anthropocene, but also to engage with those with whom we work. For those of us in academic settings this would also include our undergraduate students, not only in the general sense of our responsibilities to foster the next generation of anthropologists, but more particularly as we engage in mentor/mentee relationships with them, similar to those that we might see in graduate institutions. Even before this begins, before senior or honor's thesis or capstone projects, university professors and instructors have the opportunity to give them some meaningful field work opportunities, nevertheless there are factors which may limit our ability to do so.

From 2008-2014 at the University of Alaska Fairbanks (UAF) and from 2014 to present at Bloomsburg University of Pennsylvania (BUP) I have worked to allow students the opportunity to gain some meaningful field experiences in "their own backyard." I have found that there have been a number of challenges in doing so that I have met with varying degrees of success. In this short essay I touch on some of the limitations and challenges that I faced in incorporating hands-on work and discuss some of the techniques which have worked for me. Increasingly academic anthropologists are integrating their commitments to scholarship, research, and service. We can see as much in the work of the Digital Ethnography of Wesch [5] at Kansas State University (http://mediatedcultures.net/). My own work with students includes assessment of campus and area food systems at UAF in five classes: Food and Culture, Culture and Global Issues, Political Anthropology, Economic Anthropology, and Environmental Anthropology and Regional Sustainability. In these classes we worked to examine food deserts and food availability in Fairbanks based on existing infrastructure using a previous study on the prevalence of pseudo-foods [6]. The students worked in teams to explore food access and availability both on campus and in the larger community revealing areas of deficiency and developed reports that were later used by local and university actors to alter the food systems. The work of students was theoretically grounded in relation to what would typically be covered in a particular course. For example, the typical content delivery in Political Anthropology would include a discussion of power, agency, and social organization. These key concepts were thoroughly incorporated into classroom discussions of progress on the final project as well as the final products the students developed including reports and presentations. At BUP we worked in Environmental Anthropology and Regional Sustainability to examine two topics: environmental perceptions in the context of town-gown relationships and the legacy of anthracite coal mining in Pennsylvania with a focus on Centralia, Pennsylvania.

Table 1. Suggested Schedule in a 15 -week class

\begin{tabular}{|c|c|}
\hline Week(s) & Topic/Activity \\
\hline 1 & $\begin{array}{l}\text { a) Review of Syllabus (can be electronic review posted to LMS), Discussion of General Expectations for Group Project } \\
\text { b) Substantive Course Delivery }\end{array}$ \\
\hline 2 & $\begin{array}{l}\text { a) Online Quiz on General Anthropological Concepts from handout in Week One } \\
\text { b) Topic Selection (Centralia Mine Fire and the Legacy of Coal in Columbia County/ Environmental Perceptions and } \\
\text { Reported Behaviors of Bloomsburg University Students); } \\
\text { c) Substantive Course Delivery }\end{array}$ \\
\hline 3 & $\begin{array}{l}\text { a) Class Project Workshop (Institutional Review Board (IRB), Literature Review, Data Collection Procedures and Assign } \\
\text { Tasks) } \\
\text { b) Homework Due - Completion of IRB and Preliminary Literature Review }\end{array}$ \\
\hline $4-12$ & $\begin{array}{l}\text { a) } 15-30 \text { minutes of group work on project } \\
\text { b) Substantive Course Delivery }\end{array}$ \\
\hline 13 & Class Project Workshop (Groups Meet Extensively to Prepare Final Product for in Class Submission) \\
\hline 14 & $\begin{array}{l}\text { a) Project Paper Submission } \\
\text { b) In-Class work on Presentation Preparation } \\
\text { c) Substantive Course Delivery (as needed) }\end{array}$ \\
\hline 15 & $\begin{array}{l}\text { a) Deliver Presentation as a Group } \\
\text { b) Discussion of lesson learned, } \\
\text { c) Discussion of opportunities for further study, } \\
\text { d) Discuss opportunities for results dissemination }\end{array}$ \\
\hline
\end{tabular}




\section{Limitations and Opportunities}

There are several challenges that we must meet if we are to offer meaningful field work opportunities to our students in the context of programs that do not have an emphasis on applied work including: conflicting perceptions on the value of integration, time (in the context of a 15 -week class that meets on a given schedule each week), navigating the Institutional Review Board, varying degrees of student knowledge of general anthropological concepts and methods, general interest/buy-in to the experience, and potential issues of free-riders in the context of group projects. I will begin with the question of whether it is worth it or not to integrate applied or community-based work into substantive classes.

\subsection{Conflicting Perceptions on the Value of Integration}

Academic anthropologists approach the field and teaching from a variety or perspectives and pedagogical styles. Hence, there may be conflicting perceptions on the value of integration, from both fellow faculty and students who might be more comfortable with the "teacher as knowledge disseminator" model of education. This pedagogical approach would focus on the expertise of the instructor rather than working to cultivate a learning community focused on problem solving. This is by no means isolated in the context of teaching undergraduate anthropology. Indeed, in a larger sense, there are discussions about what comprises good teaching in the context of a liberal arts curriculum where many of us find our departments situated. Discussion have revolved around the importance of content delivery, versus imparting essential skills that will be of lasting value for both majors and non-majors alike.

Since many of the classes that I have been teaching over the past eight years have not been explicitly applied in nature but rather emphasized content delivery I have struggled to ensure a balance between the appropriate amount of content is being delivered and opportunities for field work. At the same time, I have worked to ensure that a fair amount of work assigned so as to avoid overwhelming students (avoiding 15-20 hours of commitment for one class). For those of us that make extensive use of films and lectures in our classes the increasingly available tools available through our institution's Learning Management Software (LMS) can allow us to flip some of classes so that content delivery that does not necessarily require synchronous interaction (recorded screencasts of lectures based around our course text or films accessible through YouTube, Vimeo or other web-based platform) can be shared while freeing free up class time to allow for interaction between student group members and faculty.

\subsection{Navigating the Institutional Review Board}

One of the time-based challenges that can present itself in this instance is the short time frame allowed to bring students through the necessary steps in order to apply their work in the field. Of concern for any professional anthropologist would be meeting the ethical standards of our profession in addition to the legal requirements of the "new bureaucracies of virtue" [7]. I am referring here to the box checking and compliance driven process of the Institutional Review Board whose requirements vary quite considerably from institution to institution. Indeed, in my own work on the Tohono O'odham Nation [8] as a graduate student at the University of Florida, the checks were relatively permissive as I made my way through the University of Florida's IRB and my research was approved on the Tohono O'odham Nation prior to attaining formal permission from the Tohono O'odham Nation itself. This was not the case for students at the University of Arizona who were required to attain formal permission from the Legislative Council of the Tohono O'odham Nation before their proposed research would even be considered. Of course our fields when working with undergraduate students during the fall and spring semesters are typically more geographically proximate and hence the approach of the IRB potentially more nuanced.

Nevertheless, as IRB requirements vary from institution to institution, with some being relatively permissive, one-page exemption forms that are moved through IRB within a week, to more restrictive IRBs that call for nearly 20 pages of documents needed to request an exemption with time spans of up to a month or more to attain the approval. Certainly these challenges, in terms of the amount of paperwork necessary and varying response times from the IRB can act to stymie research in the context of a 15-week course. Regardless of the timing of the IRB I have found that making clear deadlines stated on the syllabus and covered in the first day of class as a way to keep the class on track as a whole. In addition to covering the expectations in terms of a group project and timing in the first week I have carved out an entire day or even a week to define the project to be covered, complete the proposal, and make sure that the students are clear on what is being expected of them. One of the initial hurdles, depending on the institution, would be to ensure that all students coming into the class have completed the web-based CITI (Collaborative IRB Training Initiative https://www.citiprogram.org/), which in an increasing number of institutional settings is viewed as a necessary prerequisite to participate at any level of research. If students come into the class without this experience, then clearly stating the expectation of CITI completion by the end of Week 2 is essential in moving the research proposal through the IRB process.

\subsection{Varying Degrees of Knowledge of Methods, Theory and General Knowledge in Anthropology}

Students have come to my classes with varying degrees of knowledge in terms of methods and theoretical 
approaches in anthropology. In some instances, despite the class being a $400-$ level course, students take the class as their first ever anthropology class. In order to meet the initial challenge, I have developed a "cheat" sheet of approximately 10 single space pages that contains the bare minimum of what they need to know in order to understand general cultural anthropology terminology. I have found that this valuable for not only non-majors, or those new to the discipline, but also for majors as it reinforces key concepts and helps them to see the continuity of the anthropology major. This is particularly important in programs where class pre-requisites are either not in place or regularly adhered to. In addition, for those who may have had introductory courses with other professors it allows for the class as a whole to come to a shared understanding of the concepts. One thing that I have yet to institute but will likely do in the future is to have an online quiz via the LMS that is paired with the initial ten-page handout. This would test the students on the key concepts, hence ensuring that the key concepts have at least been considered or revisited by all class members.

\subsection{General Interest/Buy-in to the Experience and Issues of Free-riders}

A key component in class projects is that everyone should buy into the project. In programs where group projects are atypical, the first hurdle to overcome would be a general buy-in to the premise that group-based work versus the lone ethnographer model is not only acceptable, but also essential as anthropologist at all levels of training are called upon to work in interdisciplinary teams to address issues of practical concern. The topic of the group project is ideally something that is of enduring interest throughout the semester and potential beyond. Once the class has come to consensus on the topic(s) to be considered I have had very little to do in terms of providing motivation for the projects to be carried out as students tend to encourage one another. This is done in the context of weekly group discussion in class as they continue to coordinate, share findings with one another, and seek feedback from fellow students and myself. The class time in weeks 4-12 can also be utilized to allow for short five-minute presentations on progress to date and challenges faced between two or more groups. Further, the time can be utilized in order to collaborate and suggest potential new avenues for research when constraints might not allow for the continued fruitful pursuit of certain lines of enquiry. For example, in the case of the Centralia project students worked with one another to move away from conducing oral history interviews of former Centralia residents after they realized that these would not be likely after multiple attempts to reach out to former members of the community through personal contacts and community events yielded no scheduled interviews. These students were incorporated by their fellow group members into more fruitful avenues of research including helping to assist in archival research on Centralia, social media accounts of Centralia, and survey distribution and data analysis of coal perceptions including cost, efficiency, safety, and regulations.

This can also have the potential to extend beyond the initial class as students outside of the class become interested and seek to become involved in the project. For example, in 2014 students working on the Centralia project received some assistance from a student in another class that was interested in issues of coal and health in central Pennsylvania. Student presented aspects of both research projects at two events in the spring 2015 semester: the Pennsylvania State System of Higher Education Undergraduate Anthropology Conference and BUP's College of Liberal Arts Research Day. The class structure allowed them to pursue original work, work in teams, present in class, and present in supportive environments that will continue to fuel their intellectual development. In addition, I have shared portions of the Environmental Perception and Behaviors with BU's Green Campus Initiative and BUP's President with both offering support for continued research. Although this research at BUP and in surrounding Columbia County is still in its early stages, I am optimistic of future applied aspects as continued work on food systems by students at the University of Alaska Fairbanks led to the formation of the Chancellor's Student Food Group and initiatives to steer the direction of food procurement in campus dining facilities.

\section{Conclusions}

I hope to have provided an outline of what I believe is possible, given a variety of daunting challenges in the context of a 15-week semester class. In sum, the workflow or schedule of the class that I have recently utilized with some of the modifications that I have highlighted above can be seen in Table 1. It should be noted that in this context there is no final exam, hence further modification would be necessary in order accommodate the additional time necessary to prepare for and take a final exam. Indeed participation in the semester-long group project helps to reinforce and apply key concepts covered in readings and in-class discussions, supporting not only the delivery of content, but also its retention in a personally meaningful way.

\section{Acknowledgements}

I am grateful to the students who have worked with me on these community-based projects over the past eight years at the University of Alaska Fairbanks and Bloomsburg University of Pennsylvania. 


\section{REFERENCES}

[1] M. H. Agar, The Professional Stranger: An Informal Introduction to Anthropology. Academic Press, New York, 1980.

[2] J.M. Falcone, The Hau of Theory: The Kept-Gift of Theory Itself in American Anthropology. Anthropology and Humanism 38(2): 122-145.

[3] A. Appadurai Introduction: Place and Voice in Anthropological Theory Source: Cultural Anthropology Vol.3, No.1, 16-20

[4] E. Turner Introduction to the Art of Ethnography, Anthropology and Humanism, Vol.32, No.5, 108-116.
[5] Wesch, M. 2015 "Falling Up"- Genre-busting Digital Ethnography, Online available from http://mediatedcultures. net/videos/falling-up-genre-busting-digital-ethnography

[6] A. Wison Bringing Political Economy into the Debate on the Obesity Epidemic. Agriculture and Human Values Vol.21, No. 4, 299-312.

[7] M.A. Jacob, A. Riles The New Bureaucracies of Virtue: Introduction PoLAR: Political and Legal Anthropology Review Vol.30, No.2, 181-191.

[8] D. V. Fazzino, Continuity and Change in Tohono O'odham Food Systems: Implications for Dietary Interventions. Culture \& Agriculture Vol.30, No.1-2, 38-46. 